\title{
Cardiac Inducing RNAs (CIRs) from Human Fetal Heart Promote the Differentiation of Non-Muscle Cells to Form into Cardiomyocytes in vitro
}

ISSN: 2639-0531

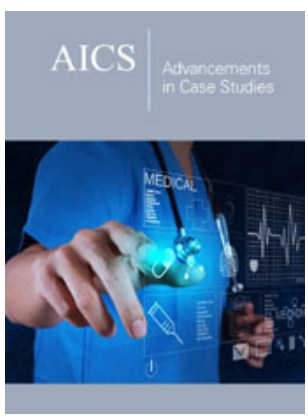

*Corresponding author: Larry F Lemanski, Texas A\&M UniversityCommerce, USA

Submission: 㘹 March 17, 2021

Published: 制 April 01, 2021

Volume 3 - Issue 1

How to cite this article: Lemanski LF, Kochegarov A, Kaveh K, Neal M, Arms A, Rodriguez YL, Hong L, et al. Cardiac Inducing RNAs (CIRs) from Human Fetal Heart Promote the Differentiation of NonMuscle Cells to Form into Cardiomyocytes in vitro. Adv Case Stud. 3(1). AICS.000553. 2021.

DOI: 10.31031/AICS.2021.03.000553

Copyright@ Lemanski LF, This article is distributed under the terms of the Creative Commons Attribution 4.0 International License, which permits unrestricted use and redistribution provided that the original author and source are credited.
Lemanski LF*1,2,3, Kochegarov A², Kaveh $\mathrm{K}^{1,2}$, Neal $\mathrm{M}^{2}$, Arms A², Rodriguez $\mathrm{YL}^{1,2}$, Hong $\mathrm{L}^{1,2}$, Equbal $\mathrm{MJ}^{1,2}$, Biswas $\mathrm{Pi}^{2}$, Biswas $\mathrm{Pr}^{2}$, Gonzalez $\mathrm{M}^{2}$, Ross-Ferguson $\mathrm{J}^{2}$, Rusk $\mathrm{J}^{2}$, Lyman-Henley $\mathrm{L}^{2}$, McRae-Kee $\mathrm{T}^{2}$, Ivory $\mathrm{C}^{2}$ and Zhao $\mathrm{Z}^{1,2}$

${ }^{1}$ Biomedical Institute for Regenerative Research, Texas A\&M University-Commerce, Commerce, TX, USA

${ }^{2}$ Department of Biological and Environmental Sciences, Texas A\&M University-Commerce, Commerce, TX, USA

${ }^{3}$ NeoHeart, LLC, Biotechnology, Commerce, TX, USA

\begin{abstract}
We have discovered a cardiac-inducing RNA (CIR) in the axolotl, Ambystoma mexicanum, (a salamander) and two cardiac inducing RNAs (CIR-6 and CIR-30) in human heart that have the ability to induce the differentiation of non-muscle cells, including induced pluripotent stem cells from human skin, mouse embryonic stem cells, and mouse fibroblasts into cardiomyocytes in vitro. Although the primary sequences of salamander and human RNAs are not homologous, their secondary structures are very similar and we believe account for their shared unique abilities to promote differentiation of non-muscle cells into definitive cardiomyocytes. We are beginning to explore the potential for repair/regeneration of cardiac muscle in vivo using mouse and rat models with induced acute myocardial infarctions (AMI) to determine if pluripotent stem cells or fibroblasts transfected with the human CIRs or CIRs alone injected into the damaged areas of the hearts can affect repair of the damaged cardiac muscle tissue and return the infarcted hearts and the AMI animal models to pre-heart-attack function again. If cardiac cells damaged in heart attacks can be replaced with living, functioning cardiomyocytes, patients with heart disease would be able to have normal heart function restored and could return to normal pre-heart-attack activity levels. Understanding how CIR transforms non-muscle cells into vigorously contracting, functional cardiac muscle and effectively replacing damaged heart cells with newly-formed cardiac muscle tissue would represent a major breakthrough in modern biology and medicine with the potential to have a significant impact on the survival rate and quality of life of millions of individuals worldwide who suffer heart attacks each year.
\end{abstract}

Keywords: Cardiac inducing RNA; Cardiomyocytes; Cardiac regeneration/repair

\section{Introduction}

The major obstacle to heart regeneration after myocardial infarction is that heart muscle cells, or cardiomyocytes, die and are replaced by fibrous scar tissue rather than new cardiac muscle cells. The fibrous scar tissue supports the damaged heart temporarily, but in the long term, it weakens the organ's overall ability to contract normally and increases the risk of a further heart attack which may lead to heart failure. This is a major reason why heart disease is the leading cause of death worldwide. According to statistics from the World Health Organization (WHO), approximately 17.9 million people per year die from cardiovascular disease, which was $31 \%$ of all deaths on Earth. Of these 17.9 million deaths, 15.2 million (or $85 \%$ ) are due to heart attack or stroke [1]. By 2030, it is estimated that failing hearts alone will kill more than 23 million people every year [2]. Currently, the only option for a severely failing heart is organ transplantation. For almost 20 years, since the early 2000s, scientists have been attempting to use stem cells from an individual's own body to grow new muscle in damaged hearts. Stem cells are capable of producing many of the various types of cells in the body, and the hope is that this will include the ability to generate new cardiomyocytes to replace those damaged by myocardial infarction or other heart disease processes. To date, there has been very limited success with being able to repair damaged areas of the heart 
following in vivo myocardial infarction or treating heart failure by using stem cells.

In our laboratory, we have discovered three unique Cardiac Inducing RNAs (CIR), one initially in the Mexican axolotl (salamander), Ambystoma mexicanum [3] and subsequently, two CIRs, derived from human fetal heart [4-8], that have the ability to turn non-muscle cells into cardiomyocytes with normal myofibrils. We undertook an approach initially developed in our laboratory to use the axolotl CIR to restore heart development and function in cardiac non-function mutant embryonic axolotls [3]. Also, we found that CIR from the axolotl, and subsequently human CIRs, have the capability of directing the fate of non-muscle mouse embryonic stem cells (ESCs), human skin-derived induced pluripotent stem cells (iPSCs) and mouse embryonic fibroblasts to express cardiac-specific proteins and differentiate into cardiomyocyte-like phenotypes in vitro [9]. We cloned and sequenced the active CIR components in the axolotl and two human CIRs. The axolotl CIR was found to be unique in the gene-bank databases [3], while the two human CIRs showed sequence homologies identified with: (1) Clone \#6 (CIR6), human exon 8 of N-sulfoglucosamine sulfhydrolase (SGSH) [4-6] and (2) Clone \#30, (CIR-30) the human COX2 superfamily of genes in the mitochondrial genome [7]. Both human CIRs turned out to be functional homologs of the axolotl (salamander) CIR, since all three of these RNAs promote cardiac myofibril formation in cardiac non-function mutant axolotl hearts as well as in mouse ESCs and human iPSCs and mouse fibroblasts in vitro [5-7,9]. Neither of the human active CIRs showed sequence homology with the original axolotl CIR or with each other, but both have secondary structures as revealed by the Gene Bee program (use provided courtesy of the Belozersky Institute, Moscow State University, Russia), that are strikingly similar to the active normal axolotl CIR, and to each other, but dissimilar to the cardiac nonfunction mutant axolotl CIR [7]. We believe that the similar secondary RNA structures account for their unique abilities to promote myofibrillogenesis and transform nonmuscle cells into cardiomyocytes [7]. (Note: we originally termed the axolotl Cardiac Inducing RNA, Myofibril Inducing RNA or MIR; we now refer to all of these RNAs, axolotl and human, as Cardiac Inducing RNA or CIR).

Our earlier published work used a cardiac-mutant axolotl (salamander) embryonic heart bioassay system [10,11]. Noncontracting mutant embryonic axolotl hearts, ordinarily lacking organized myofibrils, were placed in organ cultures with CIR derived from normal axolotl embryonic anterior endoderm or normal axolotl embryonic heart or human heart. After 48-72 hours, the mutant axolotl hearts in organ culture begin to beat rhythmically, and immunofluorescent staining with contractile protein antibodies (i.e., anti-tropomyosin, anti-cardiac specific troponin- $\mathrm{T}$, anti- $\alpha$-actinin) revealed the appearance of organized sarcomeric myofibrils of normal morphology. These studies were expanded to mouse embryonic stem cells (ESCs) and human iPSCs (induced pluripotent stem cells) derived from prepuce skin and mouse embryonic fibroblasts. Immunofluorescent staining of these cells cultured after transfection with CIR using a lipofectamine transfection system and stained with cardiac-specific troponin-T and cardiac tropomyosin clearly showed their differentiation into definitive cardiomyocytes. We also found that mutant axolotl hearts [4], mouse embryonic stem cells and human iPS cells [6] and mouse fibroblasts [9] respond to the CIR lipofectamine-induced transfection by differentiating into cardiomyocytes with cardiacspecific proteins, characteristic spindle-shaped cardiomyocyte shapes, and cardiac sarcomeric myofibrils of normal morphology in culture. Untreated controls treated with lipofectamine alone or with non-active RNAs in lipofectamine vesicles do not differentiate into cardiomyocytes.

These CIRs induce a high percentage of the stem cells and fibroblasts $(>80 \%)$ to differentiate into cardiogenic cells in vitro as determined by the expression of cardiac specific proteins (cardiac specific troponin-T, tropomyosin and $\alpha$-actinin) and characteristic cardiomyocyte-type morphologies [9]. As such, the Cardiac Inducing RNA (CIR) recently has been issued as a US Patent (Cardiac Myofibril Induction, \#10,413,617 [09/17/19]) by the United States Patent and Trademark Office (Inventors: Larry F. Lemanski [Lead Inventor]; Andrei Kochegarov [Associate Inventor]; Ashley Arms [Associate Inventor]) [12].

\section{Materials and Methods}

\section{Identifying and Preparing Synthetic Cardiac Inducing RNA (CIR)}

We constructed cDNA libraries of embryonic axolotl endoderm RNA and human fetal heart RNA and isolated plasmid DNA from 596 clones, 200 axolotl endoderm clones and 396 human fetal heart clones $[4-8,13]$. We tested the resultant synthetic RNAs' abilities to rescue mutant axolotl hearts in organ culture by turning the noncontractile mutant hearts into phenotypically normal hearts with contracting myofibrils of normal morphology. We succeeded in isolating one axolotl-derived CIR and two human CIRs that promote cardiac muscle formation in our axolotl heart bioassays [4-8].

\section{Cell culture, reagents, cell monitoring during culture period}

We culture mouse and human iPSCs and fibroblasts without feeder layers; with CIR transfection they differentiate into cardiomyocytes [3,5,9,10,13-16]. A mouse embryonic fibroblast (MEF) cell line, obtained from the American Type Culture Collection (Manassas, Virginia) (STO, ATTC®CRL-1503) was expanded and grown routinely in growth medium on DMEM medium supplemented with 10\% Fetal Bovine Serum (FBS) [13]. Cells in culture were regularly passaged when they reached full confluence. The transcription reaction mixture was prepared using a MAXIscriptW T7 Kit (Ambion \# AM1314M) and the reaction was allowed to proceed for $2 \mathrm{~h}$ at $3{ }^{\circ} \mathrm{O}$. The CIR was extracted 
and purified using ethanol precipitation and resuspended in nuclease-free water. The concentration of CIR was determined spectrophotometrically by the nanodrop method; the cells then were transfected with the active CIR using a lipofectamine transfection system at a concentration of $12 \mathrm{ng}$ CIR (RNA)/ul in Opti-MEM medium [13-15]. Culture medium was aspirated from the cells and replaced with RNA/Opti-MEM solution and incubated in a cell culture incubator (at $37{ }^{\circ} \mathrm{C}$ and $5 \% \mathrm{CO}_{2}$ ) overnight, then cultured routinely in fresh culture medium without the CIR for an additional seven days. On a daily basis the cell morphologies of the CIR-transfected and control cultures were monitored using an Olympus inverted microscope.

\section{Fixation, staining and confocal microscopy of cultured cells}

On the eighth day following CIR-transfection, the cells in the cultures were fixed, immuno-stained and processed for observation using an IX73 Inverted Olympus Fluorescent Microscope and Result an Olympus FV3000 Laser Confocal Microscope System. CIRtreated, non-inducing RNA-treated, lipofectamine-only treated or completely untreated control cells in culture were fixed in $4 \%$ paraformaldehyde for 30min, rinsed in phosphate-buffered saline (PBS) with 3\% bovine serum albumin (BSA), permeabilized in $0.1 \%$ Tween-20 and 3\% BSA, and stained overnight at $4{ }^{\circ} \mathrm{C}$ with the specific primary mouse-derived hybridoma antibodies diluted 1:75 with PBS [10-16]. The cells were then rinsed with PBS and $3 \%$ BSA for $3 \mathrm{~min}$ and stained with the secondary FITC-labeled goat anti-mouse polyclonal antibody at $4 \stackrel{\circ}{\circ} \mathrm{C}$ in a dark environment for $1 \mathrm{~h}$. The cells were immunefluorescently-stained for cardiac tropomyosin and cardiac specific troponin-T. To obtain detailed images and quantitation of the immunefluorescently-stained cardiac specific troponin-T and cardiac tropomyosin, an Olympus Fluo View® FV3000 Laser Confocal Microscope was used. Intensity of fluorescence staining detected in the confocal microscope was quantified by using ImageJ software.
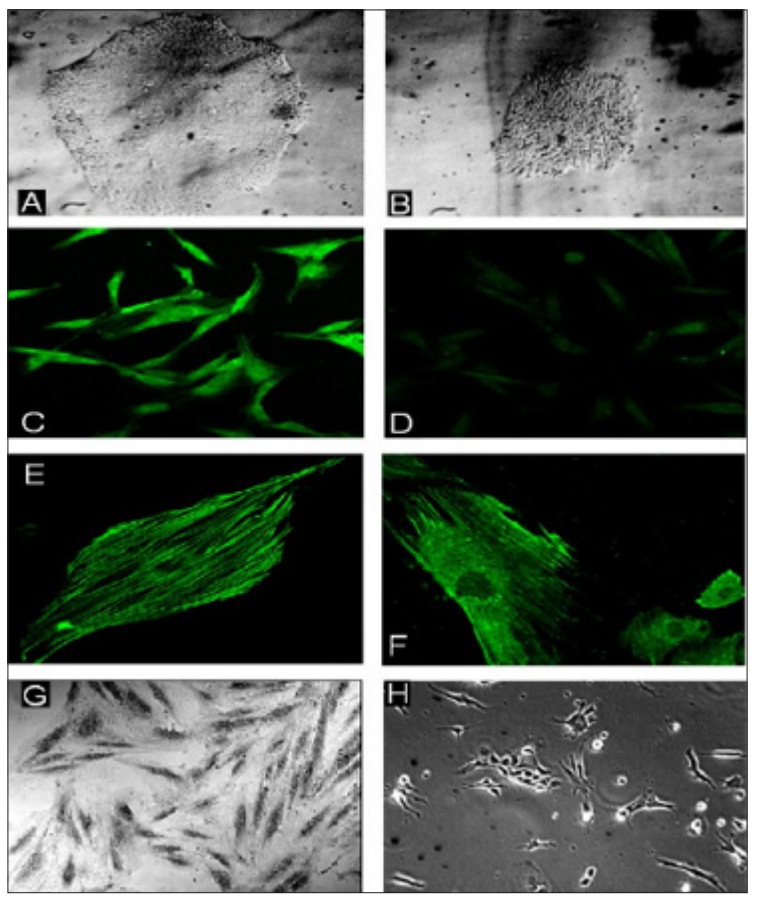

Figure 1: Colonies of (A) human iPS cells and (B) mouse ESCs in our cultures revealed by phase contrast microscopy. The rounded colonies with smooth edges indicate lack of differentiation. (C) Mouse iPSCs treated with CIR-6, cultured for 8 days, and stained with antibody to cardiac-specific troponin-T (cTnT) show significant staining for cTnT; virtually identical results are obtained with CIR -treated and stained human iPSCs. (D) Control iPS cells were cultured in the same way as in (C), except two other human heart-derived nonactive RNAs (clones \#4 and \#291) were used to treat the human iPS cells or mouse ESCs. These negative control cells showed no cTnT staining. (E) High- magnification micrograph of a human iPS cell treated with CIR- 6 and then cultured for 8 days and stained with cTnT antibody. The myofibrils have organized in parallel, and the cells are spindle shaped, characteristic of cardiac myocytes in culture. (F) High-magnification of a human iPS cell treated as in (E) but stained with antibody against alpha-actinin. Again, myofibrils are aligned,and the cells' shapes are characteristic of early cardiomyocytes in culture. (G) Mouse ESC-derived cells after CIR-6 treatment. Most show a spindle shape, indicating a cardiomyocyte lineage. $(\mathrm{H})$ Control mouse ESC-derived cells without CIR. Most of the cells show fibroblast morphologies rather than cardiac shapes. Similar results are obtained by adding nonactive RNA clones (\#4 and \#291). Note: Figures 1A, B, C, D, E, F were reproduced with permission of the authors [5]. 
Human iPSCs, mouse ESCs and mouse fibroblasts respond to CIR-6 or CIR-30 by forming $>80 \%$ cardiac troponin-T expressing and tropomyosin expressing myocardiocytes and organized myofibrils by the 8th day in culture (Figure 1D \& 2) [5,6,8]. When the cells are treated with RNAs from non-active clones fewer than $<8 \%$ form cardiac-like cells. Control iPSCs treated with non-active RNA show mainly only background staining with cardiac-specific antibodies (Figure 1D) and exhibit non-cardiac morphologies (Figure 1H), [4-8]. Mouse fibroblasts express cardiac specific troponin- $\mathrm{T}$ and tropomyosin after 4 days in culture, even while they still have characteristic branching fibroblast morphologies
(Figure 2A). As differentiation of the fibroblasts progress toward myocardial phenotypes, they lose their branched appearances and take on spindle shapes while beginning to form definitive sarcomeric myofibrils (Figure 2A, 2B, 2C). The fibroblast cultures treated with $12 \mathrm{ng} / \mathrm{ul}$ of CIR in lipofectamine vesicles show $>80 \%$ cardiac phenotypes based on expression of cardiac troponin- $\mathrm{T}$ or tropomyosin and sarcomeric myofibril staining (Figure 2D). The fibroblasts appear to transdifferentiate directly into cardiomyocytes with CIR-6 or CIR-30 transfection and do not show de-differentiation during this transition process.
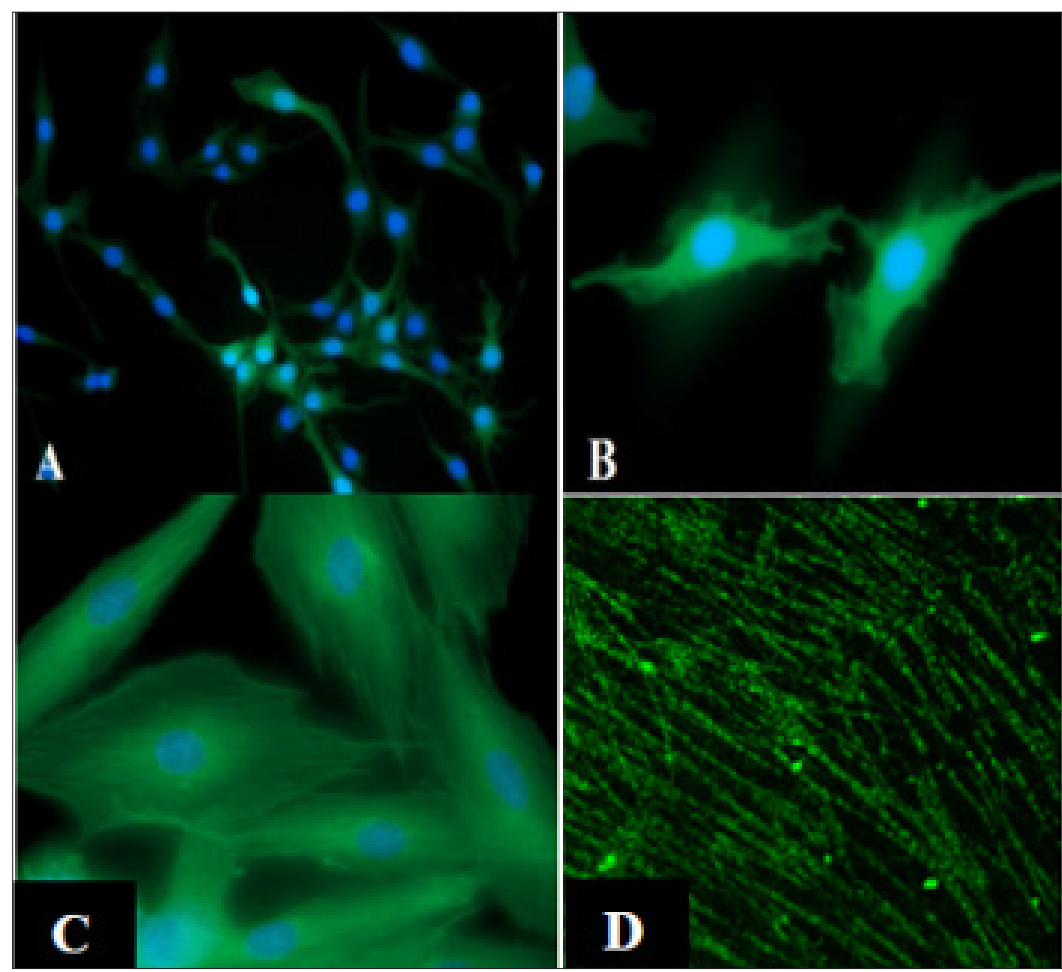

Figure 2: CIR-6 treated mouse fibroblasts stained with cardiac troponin-T (A,B,C) or sarcomeric tropomyosin (D). This higher magnification micrograph of mouse fibroblasts treated with CIR-6 and stained for sarcomeric tropomyosin shows early sarcomeric staining patterns (D).

Note: Figs. 2A, B, C, D were reproduced with permission of the authors [9].

\section{Discussion}

Transfection of human iPSCs, mouse ESCs or mouse fibroblasts with CIR-6 or CIR-30 induces expression of cardiac specific contractile protein markers as revealed by immunohistochemistry after only 4 days in culture. The fibroblasts display staining while they still show distinct fibroblast morphologies [9]. As time progresses in vitro, the CIR-transfected cultured iPSCs, ESCs and fibroblasts produce increasing quantities of cardiac troponin-T and tropomyosin and transform into in vitro cardiac cell-type morphologies. In addition, the contractile proteins (cardiac troponin-T and cardiac tropomyosin) align longitudinally as part of forming nascent myofibrils that by day 8 in culture show striated sarcomeric patterning organizations.
After 8 days in culture, $>80 \%$ of the iPSCs, ESCs and fibroblasts appear to have differentiated into definitive cardiomyocytes by using the classical definition of cardiac specific troponin-T and cardiac tropomyosin expression to define cells as "cardiac muscle cells". In controls, fewer than $<8 \%$ of the lipofectamine-only or nonactive RNA-transfected cells display cardiac-specific troponin-T or cardiac tropomyosin expression or show cardiac morphological characteristics. Thus, it is clear that the Cardiac Inducing RNAs (CIR6 and CIR-30) promote differentiation of human iPSCs and mouse ESCs into definitive cardiomyocytes in vitro. It is also evident that CIR-6 and CIR-30 induce mouse fibroblasts to transdifferentiate directly into cardiomyocytes in vitro, without any obvious dedifferentiation, pluripotent stem cell transitioning and/or re- 
differentiation phases apparent [9]. Our findings are in agreement with other studies which show that certain RNA species apparently have the ability to re-program non-muscle cells, including cardiac or dermal-derived fibroblasts toward cardiogenic lineages without going through a progenitor state $[17,18]$. Fibroblasts, iPSCs and mouse ESCs are purported to be not fully differentiated cells, thus facilitating their direct conversion into other cell types in vitro, including mesodermal cells [19]. Our results support the concept that CIR- 6 and CIR-30 stimulate a direct conversion/transdifferentiation of fibroblasts into cardiogenic cells in vitro.

Interestingly, computer analyses of the RNA secondary structures of the active human-derived CIR- 6 and CIR-30 reveal significant similarities to the Cardiac Inducing RNA (CIR) originally described for the salamander $[20,21]$ that also promotes nonmuscle cells to differentiate into cardiac muscle [7]. Thus, the human and salamander-derived CIRs, though from the different vertebrate animal classes, Mammalia versus Amphibia, appear to have highly conserved secondary RNA structures suggesting that these RNAs play major roles in all vertebrate heart development, in particular, differentiation of cardiomyocytes from nonmuscle cells during embryonic development. One of the humanderived fetal heart RNAs (CIR-6) reveals that it is a fragment of $\mathrm{N}$-sulphoglucosaminesulphohydrolase and the caspase recruitment domain family member 14 precursor gene [5] while the second CIR (CIR-30) is associated with the mitochondrial cytochrome c oxidase subunit II gene [7]. That both of these active human derived CIRs seem to be portions of larger genes may indicate that they function as non-translating epigenetic components in the cells. Based upon other recent studies, it is believed that via epigenetic processes the anti-aging gene Sirtuin 1, when repressed, is associated with agerelated human cardiovascular disease $[22,23]$. Our finding that CIR induces the transformation of non-muscle cells to form into cardiac muscle may have the potential to replace or enhance Sirtuin 1 in reducing cardiovascular disease in general. Further detailed analyses will be required to verify the intriguing possibilities of these preliminary hypotheses.

\section{Conclusion}

Our finding that greater than $>80 \%$ of the human iPSCs, mouse ESCs and mouse fibroblasts differentiate into cardiogenic cells after 8 days in culture following lipofectamine transfection of human-derived CIR- 6 or CIR-30, suggests that CIR may hold great promise for use in human medicine, in combination with an individual patient's own iPSCs or fibroblast cells for the repair of damaged myocardial infarcted tissues in vivo. By injecting the patient's own CIR transfected fibroblasts, iPSCs or CIR alone into areas of damaged heart after myocardial infarction or other disease processes, it seems possible that this approach may be able to promote the differentiation of new cardiac muscle tissue in the damaged areas of the heart and regenerate those damaged areas into normally functioning cardiac muscle again. If, indeed, this can be applied to humans, whereby a patient having had a myocardial infarction could be treated by the simple harvesting of that same patient's dermal fibroblasts (e.g., by needle biopsy), treatment of these fibroblasts in vitro with CIR-6 or CIR-30 transfection, either directly or after conversion into iPSCs, and then injection of these newly-created cardiogenic-lineage cells into the damaged area of the diseased heart might allow a patient to recover heart function and return to pre-heart-attack activity levels. This clearly would represent a major step forward in the development of a treatment and potential cure for heart failure.

Thus, the human Cardiac Inducing RNAs (CIR-6 and CIR-30) discovered in our laboratory may hold future promise for repair of damaged myocardial tissue resulting from myocardial infarction or other disease processes in human hearts. We believe that the research reviewed here has the potential for rapid translation to human clinical medicine whereby individuals who have suffered from myocardial infarction, after treatment, may be able to return to pre-heart-attack activity levels again and individuals with terminal heart failure may be able to fully recover. If this could be accomplished, cardiac transplantation would no longer be the only method available to cure heart failure, rather, treatment with CIR- 6 or CIR-30 transfected stem cells or fibroblasts might be able to replace the heart transplant treatment option by providing an effective treatment to repair/regenerate cardiac muscle damage after heart attacks and provide a complete and definitive cure for heart failure in humans.

\section{Acknowledgement}

This work was supported by NIH Grant HL061246, NSF Grant NSF RUI1121151, American Heart Association Grants 19AIREA34480001 and 15GRNT25700239 and a Texas A\&M University System Chancellor's Research Initiative Award to LFL.

\section{References}

1. https://www.who.int/en/news-room/fact-sheets/detail/ cardiovascular-diseases-(cvds)

2. https://www.heart.org/idc/groups/ahamah-public/@wcm/@sop/@ smd/documents/downloadable/ucm_470704.pdf

3. Zhang C, Jia P, Huang X, Sferrazza GF, Athauda G, et al. (2009) Myofibrilinducing RNA (MIR) is essential for sarcomeric tropomyosin expression, the organization of myofibrils and the initiation of cardiac contractile function in Mexican axolotls during development. J Biomed Sci 16(1): 81.

4. Kochegarov A, Moses A, Lian W, Hanna MC, Lemanski LF (2013) A new unique form of microRNA from human heart, microRNA-499c, promotes myofibril formation and rescues cardiac development in mutant axolotl embryos. J Biomed Sci 20(1): 20.

5. Kochegarov A, Moses-Arms A, Lemanski LF (2015a) A fetal human heart cardiac inducing RNA (CIR) promotes the differentiation of stem cells into cardiomyocytes. In vitro Cell Dev Biol Animal 51(7): 739-748.

6. Kochegarov A, Moses-Arms A, Hanna M, Lemanski LF (2015b) Micro RNA-499c induces the differentiation of stem cells into cardiomyocytes. International Archives of Medicine 8(58). 
7. Moses-Arms A, Kochegarov A, Arms J, Burlbaw S, Lian W, et al. (2015) Identification of a human mitochondrial RNA that promotes tropomyosin synthesis and myocardial differentiation. In vitro Cell Dev Biol Anim 51(3): 273-280.

8. Kochegarov A, Lemanski LF (2016) New trends in heart regeneration: A review. J Stem Cells Regen Med 12(2): 61-68.

9. Lemanski LF, Kochegarov A, Kaveh K, Neal M, Arms A, et al. (2021) Differentiation of Mouse Embryonic Fibroblasts (MEFs) into cardiomyocytes using human-derived Cardiac Inducing RNA (CIR). Stem Cells Reg Med 5(1): 1-11.

10. Zhang C, Dube D, Huang X, Zajdel RW, Bhatia R, et al. (2003) A point mutation in bioactive RNA results in RNA secondary structure alteration and failure of mutant heart correction in Mexican axolotls. Anat Embryol 206(6): 495-506

11. Rueda-de-Leon E, Kochegarov A, Lian W, Athauda G, Zhang C, et al (2011) Human heart RNA promotes tropomyosin synthesis and myofibrillogenesis in mutant axolotl hearts. MD-Medical Data 3(3): 223227.

12. Lemanski LF, Kochegarov A, Arms A (2019) Cardiac myofibril induction (Issued Patent, United States Patent and Trademark Office) Number $10,413,617$.

13. Lemanski LF, Zhang C, Kochegarov A, Moses A, Lian W, et al. (2012) Protection of mouse embryonic stem cells from oxidative stress by methionine sulfoxide reductases. In: Lushchak VI, Semchyshyn HM (Eds.), Oxidative Stress: Molecular mechanisms and biological effects; In Tech, Rijeka, Croatia, pp. 197-230.

14. Zhang C, Jia P, Jia Y, Weissbach H, Webster KA, et al. (2010) Methionine sulfoxide reductase A (MsrA) protects cultured mouse embryonic stem cells from $\mathrm{H}_{2} \mathrm{O}_{2}$-mediated oxidative stress. J Cell Biochem 111(1): 94103.
15. Zhang C, Jia P, Jia Y, Li Y, Webster KA, et al. (2011) Anoxia, acidosis and intergenic interactions selectively regulate methionine sulfoxide reductase transcriptions in mouse embryonic stem cells. J Cell Biochem 112(1): 98-106

16. Jia P, Zhang C, Jia Y, Webster KA, Huang X, et al. (2011) Identification of a truncated form of methionine sulfoxide reductase A expressed in mouse embryonic stem cells. J Biomed Sci 18(1): 46.

17. Jayawardena T, Egemnazarov B, Finch E, Zhang L, Payne JA, et al. (2012) MicroRNA-mediated in vitro and in vivo direct reprogramming of cardiac fibrobasts to cardiomyocytes. Circ Res 110(11): 1465-1473.

18. Pratico ED, Feger BJ, Watson MJ, Sullenger BA, Bowles DE, et al. (2015) RNA-mediated reprogramming of primary adult human dermal fibroblasts into c-kit (+) cardiac progenitor cells. Stem Cells Dev 24(22): 2622-2633.

19. Bar-Nur O, Gerli MFM, Di Stefano B, Almada AE, Galvin A, et al. (2018) Direct reprogramming of mouse fibroblasts into functional skeletal muscle progenitors. Stem Cell Reports 10(5): 1505-1521.

20. Lemanski LF, LaFrance SM, Erginel-Unaltuna N, Luque EA, Ward SM, et al. (1995) The cardiac mutant gene c in axolotls: cellular, developmental and molecular studies. Cell Mol Biol Res 41(4): 293-305.

21. Lemanski LF, Nakatsugawa M, Bhatia R, Erginel-Unaltuna N, Spinner B, et al. (1996) A specific synthetic RNA promotes cardiac myofibrillogenesis in the Mexican axolotl. Biochem Biophys Res Com 229(3): 974-981.

22. Martins I (2016) Anti-aging genes improve appetite regulation and reverse cell senescence and apoptosis in global populations. Adv Aging Res 5: 9-26.

23. Martins I (2017) Single gene inactivation with implications to diabetes and multiple organ dysfunction syndrome. Clin Epigenetics 3: 1-8. 